\title{
Transcultural Adaptation and Validation of Quality of Dying and Death Questionnaire in Medical Intensive Care Units in South Korea
}

\author{
Jun Yeun Cho ${ }^{1,2}$, Jinwoo Lee ${ }^{1,3}$, Sang-Min Lee ${ }^{1,3}$, Ju-Hee Park ${ }^{1,4}$, Junghyun Kim ${ }^{5}$, Youlim Kim ${ }^{1,2}$, \\ Sang Hoon Lee ${ }^{1,2}$, Jong Sun Park ${ }^{1,2}$, Young-Jae Cho ${ }^{1,2}$, Ho Il Yoon ${ }^{1,2}$, Jae Ho Lee ${ }^{1,2}$, \\ Choon-Taek Lee ${ }^{1,2}$, and Yeon Joo Lee ${ }^{1,2}$ \\ 'Department of Internal Medicine, Seoul National University College of Medicine, Seoul; \\ ${ }^{2}$ Division of Pulmonary and Critical Care Medicine, Department of Internal Medicine, Seoul National University Bundang Hospital, Seongnam; \\ ${ }^{3}$ Division of Pulmonary and Critical Care Medicine, Department of Internal Medicine, Seoul National University Hospital, Seoul; \\ ${ }^{4}$ Division of Pulmonary and Critical Care Medicine, Department of Internal Medicine, Seoul Metropolitan Government-Seoul National University Boramae Medical Center, Seoul; \\ ${ }^{5}$ Division of Pulmonary and Critical Care Medicine, Department of Internal Medicine, National Medical Center, Seoul, Korea
}

Background: Providing palliative care to dying patients in the intensive care unit (ICU) has recently received much attention. Evaluating the quality of dying and death (QODD) is important for appropriate comfort care in the ICU. This study aimed to validate the Korean version of the QODD questionnaire.

Methods: This study included decedents in the ICUs of three tertiary teaching hospitals and one secondary hospital from June 2016 to May 2017. ICU staff members were asked to complete the translated QODD questionnaire and the visual analogue scale (VAS) questionnaire within 48 hours of patient death. The validation process consisted of evaluating construct validity, internal consistency, and interrater reliability.

Results: We obtained 416 completed questionnaires describing 255 decedents. The QODD score was positively correlated with the 100 -VAS score (Pearson correlation coefficient, $0.348 ; \mathrm{P}<0.001$ ). An evaluation of the internal consistency presented favorable results (calculated Cronbach's alpha if a given item exceeded 0.8 in all items). The interrater reliability revealed no concordance between doctors and nurses.

Conclusions: The QODD questionnaire was successfully translated and validated in Korean medical ICUs. We hope further studies that use this valuable instrument will be conducted in Korea.

Key Words: attitude to death; intensive care units; medical staff; questionnaires and surveys; validation studies

\section{INTRODUCTION}

Since many patients admitted to intensive care units (ICUs) worldwide are critically ill, these units have a high mortality rate; thus, the burden of critical care is a global problem [1,2]. As a result, much attention has recently been paid to providing comfort care to dying patients in the ICU. Pain and symptom management, death preparation, achieving a sense of completion, and making decisions about treatment preferences are important factors in comfort care [3]. However,

Received on December 20, 2017 Revised on March 30, 2018 Accepted on May 3, 2018

Correspondence to: Yeon Joo Lee, Division of Pulmonary and Critical Care Medicine, Department of Internal Medicine, Seoul National University Bundang Hospital, 82 Gumi-ro 173beon-gil, Bundang-gu, Seongnam 13620, Korea

Tel: +82-31-787-7082, Fax: +82-31-787-4052, E-mail: yjlee1117@snubh.org

*No potential conflict of interest relevant to this article was reported.

(cc) This is an Open Access article distributed under the terms of the Creative Commons Attribution Non-Commercial License (http://creativecommons.org/ licenses/by-nc/4.0/) which permits unrestricted non-commercial use, distribution, and reproduction in any medium, provided the original work is properly cited. Copyright (c) 2018 The Korean Society of Critical Care Medicine 
these points have not been identified as important issues in actual practice $[4,5]$.

Providing comfort care is one of the major goals in palliative settings [6]. Maintaining an acceptable quality of life is important for dying patients in this regard. The quality of dying and death (QODD) is defined as the degree to which a person's preferences for dying and the moment of death agree with observations of how the person actually died. Patrick et al. [7] proposed a model for evaluating the QODD that consists of 31 aspects that can be rated by clinicians after a patient dies. The QODD score, an average of the ratings of each completed item, represents the degree of a patient's QODD as perceived by raters. Since the original QODD model was proposed, various versions of similar questionnaires intended for different raters (e.g., family members, medical staffers) and in different healthcare settings (e.g., outpatient, hospice, ICU) have been developed [8-10].

Many studies have been conducted in ICU settings using instruments like the QODD model, particularly in the United States and Europe. Currently, there is no validated questionnaire in South Korea to evaluate the quality of dying ICU patients. In this study, we translated the QODD questionnaire version 3.2 into Korean to validate the Korean version of the QODD questionnaire.

\section{MATERIALS AND METHODS}

\section{Study Design and Data Collection}

This study was conducted prospectively in the medical ICUs at three tertiary teaching hospitals and one secondary hospital from January 2015 to February 2016. Eligible patients were those who died in a medical ICU. Attending doctors (resident, fellow, or attending physician) and nurses caring for these patients at the time of death were asked to complete the translated QODD questionnaire within 48 hours of the death. The raters were permitted to complete the questionnaires only a limited number of times per month (a maximum of three times per month for a doctor and two times per month for a nurse).
All completed questionnaires were collected in a designated storage box in the ICU. The raters were also asked to complete a brief questionnaire about their own information (e.g., age, sex, religion, education, length of experience). The decedents' demographic characteristics (e.g., sex, age, previous medical history) and clinical information (e.g., reason for ICU admission, severity, hospital day, length of ICU stay, life support equipment, resuscitations within 24 hours) were obtained from their medical records.

\section{QODD Questionnaire}

We translated version 3.2 of the QODD questionnaire, which is designed for self-administration by a healthcare professional who cared for a patient who later died in a hospital or ICU setting [11]. We obtained permission to use the questionnaire from one of the developers prior to commencement of the study. Two experts translated the original questionnaire into Korean individually and then integrated their individual translations into a single translation. This version was retranslated into English by two additional experts, and an English language expert compared that version with the original. The final Korean version of the QODD questionnaire is included in the Supplementary Material.

The QODD questionnaire has four subcategories with items to be rated: patient experiences at the end of life (15 items), medical care at the end of life (four items), experience at the moment of death (three items), and overall ratings of care (two items). For each item, all raters were asked to evaluate the quality of each experience on a scale of 0 ("terrible") to 10 ("almost perfect"). Twenty designated items are used for calculating the QODD score, which involves summing valid ratings, dividing by the number of valid items and finally multiplying the result by 10 . The QODD score ranges from 0 to 100 , with a higher score indicating a better quality of death for the patient [11].

\section{Visual Analogue Scale Questionnaire}

The raters were also asked to complete a visual analogue scale (VAS) questionnaire to validate the QODD question- 
naire. The VAS questionnaire consisted of two items for rating: "effect on the patient's quality of death by medical treatment and the patient's experience in the ICU" and "overall quality of ICU care by medical professionals." Each item was rated on a scale from 1 (excellent) to 10 (inadequate). The mean value of valid items multiplied by 10 was designated as the "VAS score."

\section{Validation and Statistical Analysis}

All data were presented as median (range) or median (interquartile range) for continuous variables and number (percent) for categorical variables. We used IBM SPSS version 22.0 (IBM Corp., Armonk, NY, USA) for the statistical analyses. The validation process consisted of construct validity, internal consistency, and interrater reliability. Construct validity is defined as the extent to which a test assesses the concept intended to be measured [12]. To assess construct validity, we calculated the Pearson correlation coefficient between the QODD score and the 100VAS score. Internal consistency estimates were related to item homogeneity or the degree to which the items on a test jointly measure the same construct [13]. We assessed internal consistency by calculating the Cronbach's alpha if a given item was deleted. A Cronbach's alpha that exceeded 0.8 indicated good internal consistency. The interrater reliability is the extent of agreement among raters. The interrater reliability between doctors and nurses was assessed by calculating intraclass correlation coefficients (ICCs). ICCs that exceed 0.75 indicate an excellent agreement, while those below 0.40 suggest poor agreement. A $\mathrm{P}$-value $<0.05$ was considered statistically significant.

\section{Research Ethics}

This study was approved by the Institutional Review Board of each hospital. Written informed consent was obtained from medical staff at the time of each patient's death. The requirement for written consent from the patients was waived because their personal information was not used. We conducted this study in compliance with the Declaration of Helsinki.

\section{RESULTS}

\section{Baseline Characteristics of Patients and Medical Staff Members}

Of the 255 total patients with a mean age of 66.6 years, $39.6 \%$ had an underlying malignancy at the time of ICU admission (Table 1). Respiratory failure was the leading cause of ICU admission, and $20.0 \%$ of patients were admitted for post-resuscitation care. Forty-eight (18.8\%) had received cardiopulmonary resuscitation within 24 hours of death. Documented do-not-resuscitate orders were in place for 206 patients $(80.8 \%)$.

In total, 177 medical staff members completed 416 questionnaires during the 12-month study period, including 66 doctors: 52 residents (78.8\%), nine fellows (13.6\%), and

Table 1. Baseline characteristics of patients died in ICUs

\begin{tabular}{|c|c|}
\hline Variable & Total patients $(n=255)$ \\
\hline Male sex & $159(62.4)$ \\
\hline Age (yr) & $69(18-94)$ \\
\hline Malignancy & 101 (39.6) \\
\hline \multicolumn{2}{|l|}{ Reason for ICU admission } \\
\hline Sepsis & $59(23.1)$ \\
\hline Respiratory failure & $148(58.0)$ \\
\hline Heart failure & $22(8.6)$ \\
\hline Renal failure & $35(13.7)$ \\
\hline Hemorrhagic shock & $7(2.7)$ \\
\hline Post-resuscitation care & $51(20.0)$ \\
\hline APACHE II score & $27(0-71)$ \\
\hline SOFA score & $12(1-24)$ \\
\hline Hospital day & $13(0-196)$ \\
\hline Length of ICU stay (day) & $4(0-117)$ \\
\hline \multicolumn{2}{|l|}{ Life support equipment } \\
\hline Mechanical ventilator & 228 (89.4) \\
\hline Continuous renal replacement treatment & $124(48.6)$ \\
\hline Extracorporeal membrane oxygenation & $17(6.7)$ \\
\hline Others & $9(3.5)$ \\
\hline CPR within 24 hours prior to death & $48(18.8)$ \\
\hline DNR documentation prior to death & $206(80.8)$ \\
\hline
\end{tabular}


five attending physicians (7.6\%). The characteristics of the respondent ICU medical staff members are detailed in Table 2.

\section{Validation Process}

The results of the assessment of construct validity are presented in Table 3. The QODD score and subcategorical items were positively correlated with the 100-VAS score (Pearson correlation coefficient, 0.348; $\mathrm{P}<0.001$ ).

Table 4 contains the results of the assessment of internal

Table 2. Baseline characteristics of medical staffs

\begin{tabular}{|c|c|c|}
\hline Variable & Doctor $(n=66)^{a}$ & Nurse $(n=111)$ \\
\hline Age (yr) & $31(25-47)$ & $27(23-42)$ \\
\hline \multicolumn{3}{|l|}{ Sex } \\
\hline Male & $33(50)$ & $11(9.9)$ \\
\hline Female & $33(50)$ & $100(90.1)$ \\
\hline \multicolumn{3}{|l|}{ Religion } \\
\hline Catholicism & $15(22.7)$ & $14(12.6)$ \\
\hline Christianity & $15(22.7)$ & $26(23.4)$ \\
\hline Buddhism & $5(7.6)$ & $10(9.0)$ \\
\hline No religion & $31(47.0)$ & $61(55.0)$ \\
\hline \multicolumn{3}{|l|}{ Experience (mo) } \\
\hline Medical personnel & $42(24-252)$ & $24(0-216)$ \\
\hline ICU staff & $12(0-120)$ & $24(0-180)$ \\
\hline \multicolumn{3}{|l|}{ Experience of EOL care education } \\
\hline Yes & $46(69.7)$ & $27(24.3)$ \\
\hline No & $20(30.3)$ & $84(75.7)$ \\
\hline No. of completed questionnaires & 213 & 203 \\
\hline \multicolumn{3}{|c|}{$\begin{array}{l}\text { Values are presented as median (range) or number (\%). } \\
\text { ICU: intensive care unit; EOL: end of life. } \\
\text { aSixty-six doctors were participated: resident ( } n=52,78.8 \%) \text {, fellow ( } n=9 \\
13.6 \%) \text {, and attending physician ( } n=5,7.6 \%) \text {. }\end{array}$} \\
\hline
\end{tabular}

Table 3. Construct validity

\begin{tabular}{lcc}
\hline Variable & $\begin{array}{c}\text { Pearson correlation } \\
\text { coefficient }\end{array}$ & P-value \\
\hline QODD score & 0.348 & $<0.001$ \\
Patient's experience at the end of life & 0.261 & $<0.001$ \\
Medical care at the end of life & 0.332 & $<0.001$ \\
Experience at moment of death & 0.234 & $<0.001$ \\
Overall ratings of care & 0.346 & $<0.001$ \\
\hline
\end{tabular}

QODD: quality of dying and death. consistency. The calculated Cronbach's alpha exceeded 0.8 for all items, which showed good internal consistency and item heterogeneity.

Three hundred six questionnaires that were completed by both doctors and nurses and described 153 patients were used for assessing interrater reliability. the ICC for the QODD score was 0.077 , and there was no statistical significance $(\mathrm{P}=0.312)$ (Table 5).

\section{DISCUSSION}

We conducted this study because an instrument that can objectively evaluate the quality of life of dying patients should be available for use in Korean ICUs. An assessment of construct validity demonstrated a positive correlation between the 100-VAS score and the QODD score. The calculated scores for the subcategories also were generally correlated with the 100-VAS score. In the evaluation of internal consistency, all items presented high Cronbach's alpha $(>0.8)$ values. These data suggest that QODD scores calculated from the translated questionnaire could provide a useful and objective evaluation of the quality of life of dying patients, as perceived by ICU staff members in Korea. However, in terms of construct validity, there were relatively low correlations between the QODD and 100-VAS scores despite the extremely low P-value (Table 3 ). This finding may be explained by the number of extreme scores. For example, $50.8 \%$ of the raters scored 0 for the item "Being able to feed oneself" ( $47.0 \%$ in "Having a spiritual service or ceremony before death" and $42.6 \%$ in "Having one or more visits from a religious or spiritual advisor"). Many raters might not have fully understood what was being asked or lacked clarity regarding several questions of the QODD questionnaire. A revised version of the QODD questionnaire that presents the actual status of Korean ICUs should be discussed in further studies.

In the present study, a considerable number of medical staff members had no experience with comfort care education (Table 2). Currently, comfort care education has been not incorporated into standard medical school curricula in 
Table 4. Internal consistency

\begin{tabular}{|c|c|c|}
\hline Item & Rating & Cronbach's $\alpha^{\mathrm{a}}$ \\
\hline \multicolumn{3}{|l|}{ Patient's experience at the end of life } \\
\hline Having pain under control & $5(3-7)$ & 0.834 \\
\hline Having control over what is going on around you & $2(1-3)$ & 0.835 \\
\hline Being able to feed oneself & $0(0-2)$ & 0.837 \\
\hline Being able to breath comfortably & $2(1-5)$ & 0.836 \\
\hline Feeling at peace with dying & $2(1-4)$ & 0.834 \\
\hline Feeling unafraid of dying & $3(1-5)$ & 0.835 \\
\hline Being able to laugh and smile & $0(0-2)$ & 0.837 \\
\hline Keeping one's dignity and self-respect & $2(0-3)$ & 0.836 \\
\hline Spending time with family and friends & $3(1-7)$ & 0.835 \\
\hline Spending time alone & $2(0-5)$ & 0.837 \\
\hline Being touched or hugged by loved ones & $3(0-6)$ & 0.835 \\
\hline Saying goodbye to loved ones & $1(0-3)$ & 0.834 \\
\hline Clearing up bad feelings & $1(0-3)$ & 0.837 \\
\hline Having one or more visits from a religious or spiritual advisor & $1(0-4)$ & 0.836 \\
\hline Having a spiritual service or ceremony before death & $1(0-3)$ & 0.836 \\
\hline \multicolumn{3}{|l|}{ Medical care at the end of life } \\
\hline Experience of receiving mechanical ventilation & $3(2-5)$ & 0.834 \\
\hline Experience of receiving dialysis & $4(2-7)$ & 0.837 \\
\hline Receiving right amount of sedation & $6(3-8)$ & 0.834 \\
\hline Having discussed end-of-life care wishes with your doctor & $2(0-5)$ & 0.836 \\
\hline \multicolumn{3}{|l|}{ Experience at moment of death } \\
\hline Having desired people present at the time of one's death & $7(3-8)$ & 0.835 \\
\hline State at moment of death (i.e., asleep, awake, unconscious) & $4(2-7)$ & 0.833 \\
\hline Overall quality of one's dying & $3(2-5)$ & 0.834 \\
\hline \multicolumn{3}{|l|}{ Overall ratings of care } \\
\hline By all medical personnel & $7(5-8)$ & 0.837 \\
\hline By assigned doctor & $6(5-8)$ & 0.836 \\
\hline
\end{tabular}

Values are presented as median (interquartile range).

${ }^{a}$ Calculated Cronbach's alpha if a given item deleted.

Korea. Still, the ICU is a space where medical personnel are likely to encounter dying patients, and most ICU deaths are preceded by a decision to withhold or withdraw lifesustaining care $[14,15]$. Hence, comfort care education is important for medical professionals who provide comfort care. Data from a U.S. national survey showed that medical students' sense of preparedness for comfort care increases with more coursework and bedside teaching [16]. Extended opportunities for comfort care education in formal medical curricula should therefore be provided.
Table 5. Interrater reliability

\begin{tabular}{lcc}
\hline Variable & Intraclass correlation coefficient & P-value \\
\hline QODD score & 0.077 & 0.312 \\
\hline QODD: quality of dying and death.
\end{tabular}

Analysis of interrater reliability showed no concordance in QODD score between doctors and nurses (Table 5). We could not determine which factors were responsible for this finding. However, we suspect that actual differences between how doctors and nurses perceive the quality of 
life of a dying patient may be related to the amount of time spent in direct contact with the patient. Levy et al. [17] reported discordance in the QODD rating between resident physicians and nurses. A study by Breen et al. [18] also identified conflicts among staff members in $48 \%$ of cases when withdrawing life-sustaining care. In Korean medical ICUs, as is the case worldwide, nurses generally spend more time in direct contact with and closely caring for patients than do doctors. Therefore, nurses might gain a more in-depth understanding of the experiences and wishes of dying patients than doctors.

Although a good quality of death is one of the most important goals of proper comfort care, this concept is unfamiliar to Korean ICU workers. Very few studies have objectively evaluated patients' quality of death. Many dying patients still receive meaningless treatments [19], and very limited ICU visiting policies can prohibit them from spending much time with their families. Patient autonomy is often overlooked when discussing whether life-sustaining care should be provided [20]. Thus, it can be assumed that the quality of death in Korean ICUs is relatively low. Further studies that use our validated and reliable tool can demonstrate the current status of the quality of death in Korean ICUs and offer suggestions for improving the quality of death.

This study had several limitations. We did not include patients from surgical, neurologic, or other types of ICUs where comfort care is also frequently required. Samples from just four medical ICUs of acute-care-based hospitals may not be enough to validate the assessment tool for all cases. An extended validation study in various ICU settings should be conducted in the future to confirm generalizability.

In conclusion, a translated QODD questionnaire version 3.2 was successfully validated in Korean medical ICUs. We hope that further studies conducted in Korea will include this useful instrument.

\section{ORCID}

Jong Sun Park https://orcid.org/0000-0003-3707-3636

Young-Jae Cho https://orcid.org/0000-0001-6943-4462

Ho II Yoon https://orcid.org/0000-0002-5306-3692

Yeon Joo Lee https://orcid.org/0000-0002-2789-4644

\section{SUPPLEMENTARY MATERIAL}

The online-only Supplement data are available with this article online: https://doi.org/10.4266/acc.2017.00612.

\section{REFERENCES}

1. Adhikari NK, Fowler RA, Bhagwanjee S, Rubenfeld GD. Critical care and the global burden of critical illness in adults. Lancet 2010;376:1339-46.

2. Vincent JL, Marshall JC, Namendys-Silva SA, Francois B, Martin-Loeches I, Lipman J, et al. Assessment of the worldwide burden of critical illness: the intensive care over nations (ICON) audit. Lancet Respir Med 2014;2:380-6.

3. Steinhauser KE, Christakis NA, Clipp EC, McNeilly M, McIntyre L, Tulsky JA. Factors considered important at the end of life by patients, family, physicians, and other care providers. JAMA 2000;284:2476-82.

4. Hofmann JC, Wenger NS, Davis RB, Teno J, Connors AF Jr, Desbiens N, et al. Patient preferences for communication with physicians about end-of-life decisions. Ann Intern Med 1997;127:1-12.

5. Connors AF, Dawson NV, Desbiens NA, Fulkerson WJ, Goldman L, Knaus WA, et al. A controlled trial to improve care for seriously ill hospitalized patients. The study to understand prognoses and preferences for outcomes and risks of treatments (SUPPORT). JAMA 1995;274:1591-8.

6. Beckstrand RL, Callister LC, Kirchhoff KT. Providing a "good death": critical care nurses' suggestions for improving end-of-life care. Am J Crit Care 2006;15:38-45. 
7. Patrick DL, Engelberg RA, Curtis JR. Evaluating the quality of dying and death. J Pain Symptom Manage 2001;22:717-26.

8. Glavan BJ, Engelberg RA, Downey L, Curtis JR. Using the medical record to evaluate the quality of endof-life care in the intensive care unit. Crit Care Med 2008;36:1138-46.

9. Treece PD, Engelberg RA, Crowley L, Chan JD, Rubenfeld GD, Steinberg KP, et al. Evaluation of a standardized order form for the withdrawal of life support in the intensive care unit. Crit Care Med 2004;32:1141-8.

10. Curtis JR, Patrick DL, Engelberg RA, Norris K, Asp $\mathrm{C}$, Byock I. A measure of the quality of dying and death: initial validation using after-death interviews with family members. J Pain Symptom Manage 2002;24:17-31.

11. University of Washington School of Medicine. Quality of dying and death questionnaire for nurses: version 3.2. Healthcare professional after-death selfadministered questionnaire [Internet]. Seattle (WA): University of Washington School of Medicine [cited 2018 May 11]. Available from: http://depts.washington.edu/eolcare/pubs/wp-content/uploads/2011/08/ nurseqodd3_2.pdf.

12. Bagozzi RP, Yi Y, Phillips LW. Assessing construct validity in organizational research. Adm Sci Q 1991:36:42158.

13. Henson RK. Understanding internal consistency reliability estimates: a conceptual primer on coefficient alpha. Meas Eval Couns Dev 2001;34:177-89.
14. Ferrand E, Robert R, Ingrand P, Lemaire F; French LATAREA Group. Withholding and withdrawal of life support in intensive-care units in France: a prospective survey. French LATAREA Group. Lancet 2001;357:9-14.

15. Prendergast TJ, Puntillo KA. Withdrawal of life support: intensive caring at the end of life. JAMA 2002;288:2732-40.

16. Billings ME, Engelberg R, Curtis JR, Block S, Sullivan AM. Determinants of medical students' perceived preparation to perform end-of-life care, quality of end-of-life care education, and attitudes toward end-of-life care. J Palliat Med 2010;13:319-26.

17. Levy CR, Ely EW, Payne K, Engelberg RA, Patrick DL, Curtis JR. Quality of dying and death in two medical ICUs: perceptions of family and clinicians. Chest 2005;127:1775-83.

18. Breen CM, Abernethy AP, Abbott KH, Tulsky JA. Conflict associated with decisions to limit life-sustaining treatment in intensive care units. J Gen Intern Med 2001;16:283-9.

19. Bae JM, Gong JY, Lee JR, Heo DS, Koh Y. A survey of patients who were admitted for life-sustaining therapy in nationwide medical institutions. Korean J Crit Care Med 2010;25:16-20.

20. Shin K, Mok JH, Lee SH, Kim EJ, Seok NR, Ryu SS, et al. The current status of medical decision-making for dying patients in a medical intensive care unit: a singlecenter study. Korean J Crit Care Med 2014;29:160-5. 